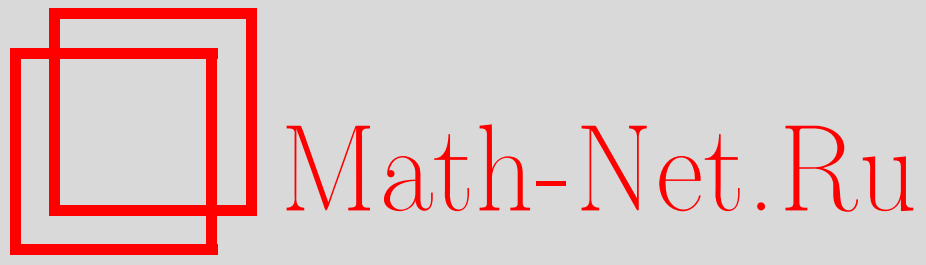

А. Ю. Окуньков, Замечание о спаривании Фурье и биномиальной формуле для многочленов Макдональда, Функи. анализ и его прил., 2002, том 36, выпуск 2, 62-68

DOI: https://doi.org/10.4213/faa191

Использование Общероссийского математического портала MathNet.Ru подразумевает, что вы прочитали и согласны с пользовательским соглашением

http://www . mathnet.ru/rus/agreement

Параметры загрузки:

IP: 54.224 .187 .69

26 апреля 2023 г., 15:48:07

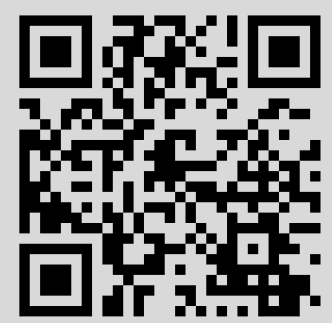




\title{
Замечание о спаривании Фурье и биномиальной формуле для многочленов Макдональда*
}

\author{
(c) 2002. А. Ю. Окуньков
}

\section{§1. Введение}

Спаривание Фурье, введенное Чередником [1], является одним из основополагающих понятий в теории многочленов Макдональда. В простейшем случае оно спаривает алгебру $\Lambda_{n}$ симметрических многочленов от $n$ переменных с алгеброй $\mathscr{D}_{n}$ коммутирующих разностных операторов Макдональда, действующих на $\Lambda_{n}[6]$. По определению

$$
\langle D, f\rangle=[D \cdot f](\widehat{0}), \quad D \in \mathscr{D}_{n}, f \in \Lambda_{n},
$$

где $\widehat{0}-$ некоторая выделенная точка. Существует естественный изоморфизм $\mathscr{D}_{n} \cong \Lambda_{n}$, который превращает спаривание (1) в квадратичную форму на $\Lambda_{n}$. Наиболее важным и полезным свойством этой формы является ее симметричность (см. $[1,6,7])$.

Основное наблюдение, сделанное в этой статье, заключается в том, что для формы (1) существует очень естественный ортогональный базис, а именно базис $\left\{I_{\mu}\right\}$, состоящий из интерполяционных многочленов Макдональда, которые интенсивно исследовались Кнопом, Ольшанским, Сахи, автором и др. (см., например, $[3,8,13]$, а также [11] и списки литературы в этих работах). Многочлены $I_{\mu}$ определяются некоторыми очень простыми многомерными интерполяционными условиями ньютоновского типа и находят множество замечательных приложений.

Ортогональность многочленов $I_{\mu}$ относительно спаривания (1), сформулированная ниже в виде теоремы 1, довольно легко следует из определений и не требует использования каких-либо нетривиальных свойств этих многочленов. Из нее сразу же вытекает симметричность спаривания (1).

Кроме того, из ортогональности многочленов $I_{\mu}$ непосредственно получается разложение по базису $\left\{I_{\mu}\right\}$ общих собственных функций операторов из алгебры $\mathscr{D}_{n}$, известных под названием симметрических многочленов Макдональда $P_{\lambda}$. Это разложение, приведенное в теореме 3 ниже, является биномиальной формулой для $P_{\lambda}$ (см. [9]). На самом деле ортогональность многочленов $I_{\mu}$ по существу эквивалентна биномиальной теореме, но, безусловно, представляется гораздо более важным, естественным и привлекательным свойством.

Биномиальная теорема из статьи [9] была распространена на более общую ситуацию, включающую другие классические корневые системы и несимметрические многочлены Макдональда (см. $[4,10,14])$. Мы в этой статье не пытаемся достичь максимально возможной общности. Наша цель, скорее, - показать, как

*Работа выполнена при поддержке NSF (грант DMS-0096246), Фонда Слоэна и Фонда Паккарда. 
основные идеи работают в простейшем нетривиальном случае обычных симметрических многочленов Макдональда. Сначала мы даже рассматриваем (почти) тривиальный одномерный случай, чтобы дать совершенно элементарную иллюстрацию того, что происходит.

Следует отметить, что существует еще один источник соотношений ортогональности для многочленов $I_{\mu}$. А именно, многочлены $I_{\mu}$ могут быть получены из симметрических многочленов Макдональда типа $B C_{n}$ (это можно очень ясно увидеть с помощью вырождения биномиальной формулы из [10] до биномиальной формулы для многочленов $\left.I_{\mu}[9]\right)$.

Эта статья основана на моей неопубликованной работе, написанной осенью 1997 г. Позднее планировалось, что она составит часть обзорной статьи об интерполяционных многочленах Макдональда, над которой мы работали вместе с Г. И. Ольшанским.

\section{§2. Простейший пример}

2.1. Сначала в качестве разминки рассмотрим одномерный случай. Предположим, что оператор $T$ действует на многочленах от $x$ по формуле

$$
[T f](x)=f(q x) \text {. }
$$

Очевидно, что одночлены $x^{n}, n=0,1,2, \ldots$, являются собственными функциями этого оператора, соответствующими собственным значениям $q^{n}$. Рассмотрим следующую билинейную форму:

$$
\langle g, f\rangle=[g(T) \cdot f](1) .
$$

В базисе $\left\{x^{n}\right\}$ эта форма имеет матрицу

$$
\left[q^{n m}\right]_{n, m=0,1, \ldots}=\left[\begin{array}{ccccc}
1 & 1 & 1 & 1 & \ldots \\
1 & q & q^{2} & q^{3} & \ldots \\
1 & q^{2} & q^{4} & q^{6} & \ldots \\
1 & q^{3} & q^{6} & q^{9} & \ldots \\
\vdots & \vdots & \vdots & \vdots & \ddots
\end{array}\right],
$$

и ясно, что она симметрическая.

Очевидно также, что

$$
x^{*}=T, \quad T^{*}=x,
$$

где $x$ обозначает оператор умножения на соответствующую независимую переменную, а «звездочка» - сопряженный оператор относительно формы (2). Ясно, что (3) определяет антиизоморфизм $q$-гейзенберговой алгебры, порожденной операторами $T$ и $x$ (подчиненными соотношению $T x=q x T$ ), который заслуживает, чтобы его назвали преобразованием Фурье.

2.2. Теперь рассмотрим многочлен

$$
I_{n}=(x-1)(x-q) \cdots\left(x-q^{n-1}\right), \quad n=0,1, \ldots,
$$

удовлетворяющий следующим интерполяционным условиям Ньютона:

$$
\begin{aligned}
I_{n} & \equiv x^{n} \quad \bmod \left\{x^{m}\right\}_{m<n}, \\
I_{n}\left(q^{m}\right) & =0, \quad 0 \leqslant m<n .
\end{aligned}
$$


ПРЕДЛОЖеНИЕ 1. Многочлены $I_{n}$ ортогональны относительно формы (2), а именно

$$
\left\langle I_{n}, I_{m}\right\rangle=\delta_{n, m} I_{n}\left(q^{n}\right) .
$$

ДоКАЗАТЕЛЬСтво. В наших рассуждениях мы будем намеренно избегать использования симметричности формы (2), поскольку в общем случае мы хотим получить аналогичную симметричность в качестве следствия.

Из определения (2) ясно, что

$$
\left\langle x^{n}, f\right\rangle=f\left(q^{n}\right),
$$

и так как $T \cdot x^{n}=q^{n} x^{n}$, то также выполняется равенство

$$
\left\langle g, x^{n}\right\rangle=g\left(q^{n}\right) .
$$

Из условия (6) теперь следует, что

$$
\left\langle x^{m}, I_{n}\right\rangle=\left\langle I_{n}, x^{m}\right\rangle=0, \quad m<n,
$$

и ясно также, что

$$
\left\langle x^{n}, I_{n}\right\rangle=\left\langle I_{n}, x^{n}\right\rangle=I_{n}\left(q^{n}\right) .
$$

Тогда свойство (5) завершает доказательство.

Из равенств (7) и (8) непосредственно вытекает следующее разложение:

$$
x^{n}=\sum_{m} \frac{\left\langle x^{n}, I_{m}\right\rangle}{\left\langle I_{m}, I_{m}\right\rangle} I_{m}(x)=\sum_{m} \frac{I_{m}\left(q^{n}\right) I_{m}(x)}{I_{m}\left(q^{m}\right)} .
$$

Это интерполяция Ньютона одночлена $x^{n}$ с узлами $1, q, q^{2}, \ldots$, а также пример $q$-биномиальной теоремы.

\section{§3. Симметрические многочлены Макдональда}

3.1. Перейдем теперь к многочленам от $n$ переменных $x_{1}, \ldots, x_{n}$. Положим

$$
\left[T_{i} f\right]\left(x_{1}, \ldots, x_{n}\right)=f\left(x_{1}, \ldots, q x_{i}, \ldots, x_{n}\right) .
$$

Пусть $t-$ дополнительный параметр. Введем, следуя Макдональду [6], операторы

$$
D_{k}=t^{k(k-1) / 2} \sum_{|S|=k} d_{S}(x) \prod_{i \in S} T_{i},
$$

где сумма берется по подмножествам $S \subset\{1, \ldots, n\}$, которые состоят из $k$ элементов, и

$$
d_{S}(x)=\prod_{i \in S, j \notin S} \frac{t x_{i}-x_{j}}{x_{i}-x_{j}} .
$$

3.2. Операторы $D_{k}$ являются коммутирующими и преобразуют симметрические многочлены в симметрические многочлены. В базисе из мономиальных симметрических функций они действуют «треугольным образом», а именно,

$$
D_{k} \cdot m_{\lambda} \equiv e_{k}(\widehat{\lambda}) m_{\lambda} \quad \bmod \left\{m_{\mu}\right\}_{\mu<\lambda},
$$

где $\lambda=\left(\lambda_{1} \geqslant \lambda_{2} \geqslant \ldots \geqslant \lambda_{n}\right)-$ некоторое разбиение,

$$
m_{\lambda}=x_{1}^{\lambda_{1}} \cdots x_{n}^{\lambda_{n}}+\text { перестановки }
$$


- соответствующая мономиальная симметрическая функция, $e_{k}=m_{\left(1^{k}\right)}$ есть $k$-я элементарная симметрическая функция, $\widehat{\lambda}$ обозначает точку

$$
\widehat{\lambda}=\left(q^{\lambda_{1}} t^{n-1}, q^{\lambda_{2}} t^{n-2}, \ldots, q^{\lambda_{n-1}} t, q^{\lambda_{n}}\right),
$$

а $\mu<\lambda$ означает отношение доминирования на разбиениях:

$$
\mu \leqslant \lambda \Longleftrightarrow\left(\begin{array}{c}
\mu_{1} \leqslant \lambda_{1}, \\
\mu_{1}+\mu_{2} \leqslant \lambda_{1}+\lambda_{2}, \\
\cdots \\
\ldots+\mu_{n}=\lambda_{1}+\cdots+\lambda_{n}
\end{array}\right) .
$$

Общие собственные функции $P_{\lambda}$ операторов $D_{k}$,

$$
D_{k} \cdot P_{\lambda}=e_{k}(\widehat{\lambda}) P_{\lambda}
$$

нормированные условием

$$
P_{\lambda} \equiv m_{\lambda} \bmod \left\{m_{\mu}\right\}_{\mu<\lambda},
$$

называются симметрическими многочленами Макдональда.

3.3. Пусть $\Lambda_{n}$ обозначает алгебру симметрических многочленов от $n$ переменных. Из равенства (9) следует, что отображение

$$
D: \Lambda_{n} \ni e_{k} \mapsto D_{k}
$$

продолжается до гомоморфизма алгебр, такого, что

$$
D(g) \cdot P_{\lambda}=g(\widehat{\lambda}) P_{\lambda}, \quad g \in \Lambda_{n} .
$$

Теперь определим, следуя Череднику [1], такое спаривание Фурье:

$$
\langle g, f\rangle=[D(g) \cdot f](\widehat{0}), \quad f, g \in \Lambda_{n} .
$$

Это аналог формы (2). Ясно, что

$$
\langle h g, f\rangle=\langle g, D(h) f\rangle .
$$

Другими словами, $D(h)=h^{*}$, где $h$ рассматривается как оператор умножения, а «звездочка» обозначает его преобразование Фурье, т. е. оператор, сопряженный к нему относительно спаривания (12). Из равенства (9) следует, что спаривание (12) превращает нормированную собственную функцию

$$
N_{\lambda}=\frac{P_{\lambda}}{P_{\lambda}(\widehat{0})}
$$

в $\delta$-функцию в точке $\widehat{\lambda}$, а именно

$$
\left\langle g, N_{\lambda}\right\rangle=g(\widehat{\lambda})
$$

3.4. Теперь наша цель - построить явный ортогональный базис для квадратичной формы (12). Составляющие этот базис многочлены, как и многочлены (4), будут некоторыми интерполяционными многочленами Ньютона.

Пусть $\triangleleft-$ произвольное линейное упорядочение на множестве разбиений $\lambda$, совместимое как с упорядочением разбиений по их размеру $|\lambda|$, так и с отношением доминирования, когда речь идет о разбиениях одного и того же числа. 
Определим интерполяционные многочлены Макдональда $I_{\mu}$ с помощью следующих обобщений условий (5) и (6):

$$
\begin{aligned}
I_{\mu} & \equiv m_{\mu} \quad \bmod \left\{m_{\lambda}\right\}_{\lambda \triangleleft \mu}, \\
I_{\mu}(\widehat{\lambda}) & =0, \quad \lambda \triangleleft \mu .
\end{aligned}
$$

Существование и единственность таких многочленов для $q$ и $t$ общего положения очевидным образом вытекает из их существования и единственности для $t=1$, а это устанавливается совсем просто.

3.5. Можно показать (см., например, $[3,8,13]$, а также [11]), что многочлены $I_{\mu}$ не зависят от выбора упорядочения $\triangleleft$ и обладают гораздо более сильным свойством сверхобнуления

$$
I_{\mu}(\widehat{\lambda})=0, \quad \mu \not \subset \lambda .
$$

В силу биномиальной формулы (22) отсюда вытекает следующее усиление условия (15):

$$
I_{\mu} \equiv P_{\mu} \quad \bmod \left\{P_{\lambda}\right\}_{\lambda \subset \mu} .
$$

Свойство сверхобнуления (17), однако, в дальнейшем нам не понадобится, что позволяет применять наши соображения в ситуациях, когда аналог свойства (17) места не имеет.

3.6. Наш основной результат заключается в следующем:

Теорема 1. Многочлены $I_{\mu}$ ортогональны относительно спаривания Фурье (12).

Непосредственным следствием этой теоремы является такой центральный результат теории многочленов Макдональда:

СледствиЕ 1 (Корнвиндер [6]). Спаривание Фурье (12) является симметрическим.

На самом деле Корнвиндер доказал эквивалентное свойство симметричности, а именно следующую симметрию между меткой и аргументом для нормированных многочленов (13):

$$
N_{\lambda}(\widehat{\mu})=N_{\mu}(\widehat{\lambda}) .
$$

Многочисленные приложения этой симметрии, такие, как, например, формулы типа Пиери для многочленов Макдональда можно найти в $[1,6,7]$.

3.7. Доказательство теоремы 1 проходит в два этапа. Сначала мы утверждаем, что

$$
\left\langle I_{\mu}, I_{\lambda}\right\rangle=0, \quad \mu \triangleright \lambda .
$$

В самом деле, согласно формулам (15), (10) и (14), это эквивалентно соотношению

$$
\left\langle I_{\mu}, N_{\lambda}\right\rangle=I_{\mu}(\widehat{\lambda})=0, \quad \mu \triangleright \lambda,
$$

которое действительно справедливо в силу условия (16).

3.8. Теперь докажем, что

$$
\left\langle I_{\mu}, I_{\lambda}\right\rangle=0, \quad \mu \triangleleft \lambda .
$$

В силу свойства (15) это эквивалентно доказательству того, что $\left\langle m_{\mu}, I_{\lambda}\right\rangle=0$, если $\mu \triangleleft \lambda$. Поскольку

$$
e_{\mu} \stackrel{\text { def }}{=} e_{\mu_{1}} \cdots e_{\mu_{n}} \equiv m_{\mu} \quad \bmod \left\{m_{\nu}\right\}_{\nu<\mu}
$$


достаточно доказать, что

$$
\left\langle e_{\mu}, I_{\lambda}\right\rangle=0, \quad \mu \triangleleft \lambda .
$$

В силу определения (12) это эквивалентно равенству

$$
\left[D_{\mu} \cdot I_{\lambda}\right](\widehat{0})=0, \quad D_{\mu}=D_{\mu_{1}} \cdots D_{\mu_{n}},
$$

которое мы теперь будем доказывать.

3.9. Свойство

$$
\left(\begin{array}{l}
\lambda_{i}=\lambda_{i+1}, \\
i \notin S, i+1 \in S
\end{array}\right) \Longrightarrow d_{S}(\widehat{\lambda})=0
$$

является для операторов $D_{k}$ ключевым. Из него вытекает, что

$$
\left[D_{k} \cdot f\right](\widehat{\lambda})=\sum_{\nu / \lambda=\text { вертикальная } k \text {-полоса }} d_{S(\nu, \lambda)}(\widehat{\lambda}) f(\widehat{\nu}),
$$

где $S(\nu, \lambda)=\left\{i, \nu_{i}>\lambda_{i}\right\}$. Следовательно,

$$
\left[D_{\mu} \cdot f\right](\widehat{0})=\sum_{\nu<\mu} c_{\mu, \nu} f(\widehat{\nu})
$$

для некоторых коэффициентов $c_{\mu, \nu}$. Аналогичное свойство можно установить в более общей ситуации, например, для несимметрических многочленов Макдональда [2].

Ясно, что формула (20) вместе с (16) влечет за собой равенство (19), и это завершает доказательство теоремы 1.

3.10. Теорему 1 можно усилить следующим образом:

Теорема 2. Имеют место равенства

$$
\left\langle I_{\mu}, I_{\nu}\right\rangle=\delta_{\mu, \nu} I_{\mu}(\widehat{\mu}) P_{\mu}(\widehat{0})=\delta_{\mu, \nu} c_{\mu, \mu} I_{\mu}(\widehat{\mu}) .
$$

В частности, это показывает, что $P_{\mu}(\widehat{0})=c_{\mu, \mu}$. Подставив явное выражение для $c_{\mu, \mu}$, можно убедиться, что последнее равенство эквивалентно известной формуле для $P_{\mu}(\widehat{0})$ (см. [6]).

ДоКАЗАТЕЛЬСтвО. С помощью тех же рассуждений, что и в разд. 3.7 , мы получаем, что

$$
\left\langle I_{\mu}, I_{\mu}\right\rangle=\left\langle I_{\mu}, P_{\mu}\right\rangle=I_{\mu}(\widehat{\mu}) P_{\mu}(\widehat{0}) .
$$

С другой стороны, рассуждения, аналогичные проведенным в разд. 3.8, показывают, что

$$
\left\langle I_{\mu}, I_{\mu}\right\rangle=\left[D_{\mu} \cdot I_{\mu}\right](\widehat{0})=c_{\mu, \mu} I_{\mu}(\widehat{\mu}) .
$$

3.11. Из теоремы 2 вытекает следующая интерполяционная формула Ньютона:

$$
f=\sum_{\mu} \frac{\left\langle I_{\mu}, f\right\rangle}{\left\langle I_{\mu}, I_{\mu}\right\rangle} I_{\mu}, \quad f \in \Lambda_{n} .
$$

В частности, применяя ее к $N_{\lambda}$ и используя формулу (14), мы получаем такое разложение (в котором явно указываем переменную $x$, чтобы подчеркнуть симметрию между меткой и аргументом): 
ТЕОРема 3 (биномиальная формула [9]). Имеет место разложение

$$
N_{\lambda}(x)=\sum_{\mu} \frac{I_{\mu}(\widehat{\lambda}) I_{\mu}(x)}{\left\langle I_{\mu}, I_{\mu}\right\rangle} .
$$

Из свойства (17) следует, что в этом разложении в действительности появляются только те $\mu$, которые удовлетворяют условию $\mu \subset \lambda$.

\section{ЛИТЕРАТУРА}

1. Cherednik I. Macdonald's evaluation conjectures and difference Fourier transform. Invent. Math., 122, No. 1, 119-145 (1995).

2. Cherednik I. Nonsymmetric Macdonald polynomials. Intern. Math. Res. Notices, 483-515 (1995).

3. Knop F. Symmetric and non-symmetric quantum Capelli polynomials. Comment. Math. Helv., 72, 84-100 (1997).

4. Knop F. Combinatorics and invariant theory of multiplicity free spaces. Preprint math.RT/0106079.

5. Knop F., Sahi S. Difference equations and symmetric polynomials defined by their zeros. Internat. Math. Res. Notices, No. 10, 473-486 (1996).

6. Macdonald I. G. Symmetric Functions and Hall Polynomials, 2nd ed., Oxford University Press, 1995. [Имеется перевод 1-го издания: Макдональд И. Симметрические функции и многочлены Холла. Мир, М., 1985.]

7. Macdonald I. G. Symmetric Functions and Orthogonal Polynomials. Dean Jacqueline B. Lewis Memorial Lectures presented at Rutgers University, New Brunswick, NJ, University Lecture Series, Vol. 12, Amer. Math. Soc., Providence, RI, 1998.

8. Okounkov A. (Shifted) Macdonald polynomials: $q$-integral representation and combinatorial formula. Compositio Math., 112, No. 2, 147-182 (1998).

9. Okounkov A. Binomial formula for Macdonald polynomials and applications. Math. Res. Lett., 4, No. 4, 533-553 (1997).

10. Okounkov A. BC-type interpolation Macdonald polynomials and binomial formula for Koornwinder polynomials. Transform. Groups, 3, No. 2, 181-207 (1998).

11. Okounkov A. Combinatorial formula for Macdonald polynomials, Bethe Ansatz, and generic Macdonald polynomials. Preprint math.QA/0008094.

12. Okounkov A., Olshanski G. Shifted Jack polynomials, binomial formula, and applications. Math. Res. Lett., 4, No. 1, 69-78 (1997).

13. Sahi S. Interpolation, integrality, and a generalization of Macdonald's polynomials. Internat. Math. Res. Notices, No. 10, 457-471 (1996).

14. Sahi S. The binomial formula for nonsymmetric Macdonald polynomials. Duke Math. J., 94, No. 3, 465-477 (1998).

University of California at Berkeley,

Department of Mathematics

Поступило в редакцию

e-mail: okounkov@math.berkeley.edu

5 июля 2001 г. 